\title{
The Role of Learning the Japanese Kuku Multiplication Chant in Simple Arithmetic Operations
}

\author{
Hiroyasu Ito, Namiko Kubo-Kawai, Nobuo Masataka \\ Primate Research Institute, Kyoto University, Inuyama, Japan. \\ Email: masataka@pri.kyoto-u.ac.jp \\ Received April 5 $5^{\text {th }}, 2011$; revised May $7^{\text {th }}, 2011$; accepted May $16^{\text {th }}, 2011$.
}

\begin{abstract}
In Japanese primary schools, children are required to learn the kuku ("nine nines") method of multiplication during the formal course of mathematics. When learning, they are taught to recite it as though reciting a Chinese poem or chanting. In the present study, we undertook an experiment designed to examine the role of learing the Japanese kuku multiplication chant in arithmetic operations by requiring the participants to solve the three types of simple arithmetic problems. In each problem presentation, an equation of simple addition (e.g., 3 (three) added to 4 (four) makes 7 (seven)), of simple multiplication (e.g., 3 (three) multiplied by 4 (four) is 12 (twelve)), or of kuku (e.g., 3 (three) 4 (four) 12 (twelve)) was auditorily presented with either the addend or augend in the addition, or the multiplicand or multiplier in the multiplication or kuku always being acoustically masked by peep sounds so that the participants did not hear the numbers masked. Comparison of the latency to their answer across the three types of problems revealed that as a consequence of learning kuku, a learner could produce the answers for the arithmetic multiplication problems as well as the answers for the kuku problems relatively more easily as compared to the arithmetic addition problems. Implications of the results are argued with reference to the cognitive load theory, a theory of learning and education which underwent substantial development and expansion during last two decades.
\end{abstract}

Keywords: Kuku, Mathematics, Arithmetic Operation, Number Sense, Cognitive Load Theory

\section{Introduction}

Numbers might be considered a very recent cultural invention in the evolution of the human species. Indeed, number words and digits arise from the specifically human and evolutionarily recent ability to create and mentally manipulate complex symbols. However, the sense of numbers is older. A sensitivity to numerical properties of the world is present even in nonhuman species (Dehaene, 1997; Dehaene, Dupoux, \& Mehler, 1990; Gallsitel \& Gelman, 1992). For instance, lionesses with their prides on the Serengeti Plains, Tanzania, decide to attack back only when the number of defenders is superior to the number of intruders. When deciding to drive the intruders off or not, they evaluate the number of intruders just by hearing the roars produced (McComb, Packer, \& Pusey, 1994). Altogether, previous evidence consistently indicates that this mental magnitude representation supports the more complex symbolic numerical capabilities developed by humans alone.

Although such basic preverbal "number sense" provides a foundation for formal mathematics by performing arithmetic operations on approximate number representations, the developmental process of the capability of formal mathematical performance would be environmentally modulated across the variations of linguistic cultures in the world. Nevertheless, experimental evidence revealing such variations is still limited except for that with deaf people who acquired sign languages as their first languages (Bull, Marshark, \& Baltto-Vallee, 2005; Frosted, 1996; Heiling, 1995; Nunes \& Moreno, 2002). In order to pursue this issue, in the present study, we investigated the role of learning the Japanese kuku multiplication chant in arithmetic operations in the formal mathematics.

In Japanese primary schools, children are required to memorize the kuku ("nine nines") method of multiplication during the formal course of mathematics. When learning, they are taught to recite it as though reciting a Chinese poem or chanting. The digit names are often modified to maintain the rhythm, analogous to the phenomenon to recitation of a rhythmic poetry. As the learning proceeds, all children, unless mentally retarded, become capable of reciting it from memory, and the stored knowledge, as verbal quantitative information, might serve as a foundation for the formal mathematics they will learn later. Assuming such possibility would one to reason the learning of the kuku to be particularly helpful to children with dyscalculia, which may affect as much as $5 \%$ of the total population (Sousa, 2008). In fact, the history of kuku can be traced back more than 1,000 years (Lancy, 1983), during which, it might have been traditionally transmitted as a powerful convention of creative education to support people experiencing specific learning difficulties. Nonetheless, such potential creativity of the kuku still remains novel in Western countries. In order to reveal that experimentally, the present investigation was conceived.

As a first step to explore the possibility noted above, we undertook an experiment designed to examine the role of learning the kuku in arithmetic operations by requiring the adult participants to solve simple arithmetic problems. As our intent is to investigate the role of learning the kuku in subsequent formal mathematics development, it is certainly ideal to select primary school students as the participants. It is also necessary to trace the performance of these primary school students in formal mathematics as a later stage. As a preliminary stage of such future research, the present study was conducted. The findings 
revealed are argued with reference to the cognitive load theory (CLT) because it is the most prevalent theory that explains how the human cognitive architecture, in particular our memory system, impacts learners' performance (Sweller, 1988; Paas, Renkl, \& Sweller, 2003). According to CLT, there are three types of loads, namely intrinsic cognitive load, extraneous cognitive load, and germane cognitive load, each of which has different implications for learning. In this article, we attempted to discuss the findings in terms of such conceptual framework of CLT.

\section{Methodology}

In each problem presentation, in the present study, an equation of simple addition (e.g., 3 (three) added to 4 (four) makes 7 (seven)), of simple multiplication (e.g., 3 (three) multiplied by 4 (four) is 12 (twelve)), or of kuku (e.g., 3 (three) 4 (fours) 12 (twelve)) was auditorily presented with either the addend or augend in the addition, or the multiplicand or multiplier in the multiplication or kuku always being acoustically masked by peep sounds so that the participants did not hear the numbers masked. We asked them to answer the numbers which were masked by the sounds as quickly as possible, and we compared the latency to their answer across these three types of problems. We hypothesized that as a consequence of learning kuku, the learner would find answers for the arithmetic multiplication problems relatively easily as compared to the arithmetic addition problems.

The participants were 20 adult (10 men and 10 women) in their twenties (mean age: 22.10 years, SD: 1.02 ) who had acquired Japanese as their first language. They had all been educated in the Japanese formal education system since the beginning of the entrance into kindergarden at least until the completion of graduation from senior high school. All were healthy and typically-developed. They had learned kuku in primary school and had memorized it.

During the experiment, each participant was seated in an attenuation chamber and wore a headphone. Each problem was presented auditorily through the headphone using notebook computer. Total of 20 equations of arithmetic addition, using single digits (referred to as "addition problems" below), 20 equations of arithmetic multiplication, using single digits ("multiplication problems"), and 20 phrases of kuku ("kuku problems") were presented to each participant. In 10 of the 20 addition problems, the addend was acoustically masked by peepsounds whereas the augend was masked in the remaining 10 . In each 10 of the 20 multiplication or of the 20 kuku problems, the multiplicand was masked while the multiplier was masked in the remaining 10 . The order of a total of 60 presentations was randomized. Inter-problem intervals were $5 \mathrm{sec}$. The participants were asked to answer each problem as rapidly as possible by pressing the number key located on a table near by the participant corresponding to the answer. The behavioral measure was the interval between the end of the presentation and the onset of the pressing of the button (RT; reaction time).

\section{Results}

Results of our examination of the effects of learning kuku on arithmetic operations are summarized in Figure 1. When an addition problem was presented, mean (SD) RT was 6.44 (0.28) $\mathrm{s}$ when the augend was masked and $6.50(0.27) \mathrm{s}$ when the addend was masked. When a multiplication problem was presented, mean (SD) RT was 6.17 (0.36) s when the multiplicand was masked and $6.10(0.37) \mathrm{s}$ when multiplier was masked. When the kuku problem was presented, mean RT (SD) was $6.14(0.32) \mathrm{s}$ when the multiplicand was masked and $5.96(0.39)$ $\mathrm{s}$ when the multiplier was masked. A two-factor within-subject analysis of variance revealed significant main effects for type of problems $(\mathrm{F}(2,38)=61.19, p<.001)$ and for the position of the masking sound $(\mathrm{F}(1,19)=5.82, p<.05)$. There was also a significant interaction between the factors $(\mathrm{F}(2,38)=10.56, p$ $<.001$ ). Multiple comparison analyses revealed that RT when the addition problems were presented was significantly longer than that when multiplication problems were presented $(p<.01)$ and that when kuku problems were presented $(p<.001)$. When the multiplicand was masked, RT was significantly longer that that when the multiplier was masked in both the multiplication problems $(p<.01)$ and in the kuku problems $(p<.001)$. However, in the addition problems, RT did not differ between when the augend was masked or when the addend was masked $(p>.10)$. Before you begin to format your paper, first write and save the content as a separate text file. Keep your text and graphic files separate until after the text has been formatted and styled. Do not use hard tabs, and limit use of hard returns to only one return at the end of a paragraph. Do not add any kind of pagination anywhere in the paper. Do not number text heads-the template will do that for you.

\section{Discussion}

The RT required to answer each type of the presented arithmetic problems would mostly be determined dependent upon the degree to which a working memory load is involved in the task (Baddeley, 2000). When solving problems, the task more or less puts requiring on temporarily holding and manipulating task-relevant information as a consequence of previous learning experiences (Dehaene et al., 2003). Once the information is stored, control process within working memory occurs by enabling the coordination, transformation, and integration of stored information (Masataka et al., 2007). According to this reasoning, the fact itself would not be astonishing that the RT for solving the kuku problems was much shorter than that for solving the addition problems. This is a typical example of learning

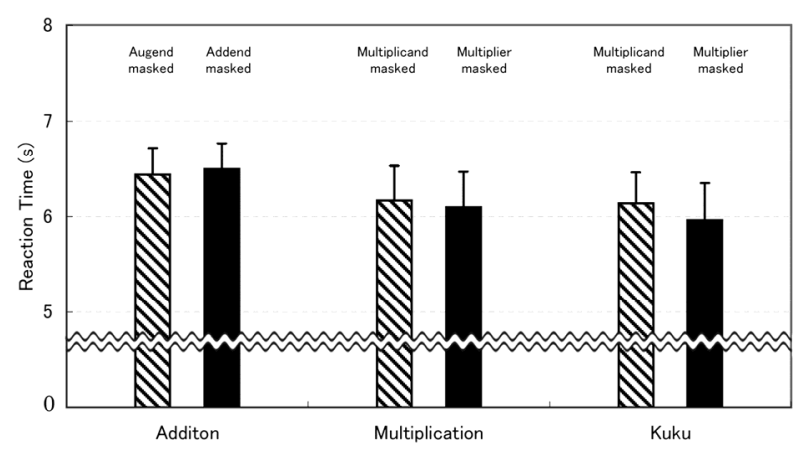

Figure 1.

Results of the examination of the effects of learning kuku on arithmetic operations. 
process outlined by CLT (Sweller, 1988; Paas, Renkl, \& Sweller, 2003), in which each relevant element of high-element interactivity material that enables learning can be learned individually, but they cannot be understood until all of the elements and their interactions are processed simultaneously. Such element interactivity is the drive of the first category of cognitive load in CLT, which is referred to as intrinsic cognitive load because demands on working memory capacity imposed by element interactivity are intrinsic to the material being learned. Different materials differ in their levels of element interactivity and therefore intrinsic cognitive load, and they cannot be altered by instructional manipulations.

In fact, working memory, in which all conscious cognitive processing occurs, is capable of handling only a very limited number of novel interacting elements. While this number is far below the number of interacting elements that occurs in most areas of human intellectual activity, long-term memory provides humans with the ability to vastly expand this processing ability. This memory store can contain vast numbers of schemas that are cognitive constructs which incorporates multiple elements of information into a single element with a specific function. In this regard, the fact should be noticeable that the RT for solving the multiplication problems recorded in the present study was approximately equivalent to that for solving the kuku problems rather than to that for solving the addition problems. This strongly indicates that the participants utilized their knowledge of kuku to obtain the answers for the multiplication problems. No doubt, this knowledge is stored in longterm memory.

CLT explains that schemas can be brought from long-term memory to working memory. While working memory might, for example, only deal one element that can be handled easily among a large number of interacting elements, their incorporation in a schema reduces the load of working memory. It is by this process that human cognitive architecture handles complex material that appears to exceed the capacity of working memory and that learning the Japanese kuku multiplication chant becomes an effective aid to performing arithmetic operations.

\section{Acknowledgements}

The present study was supported by a grant-in-aid from the Ministry of Education, Science, Sports and Culture, Japanese Government (\#20243034) as well as by Global COE Research Program (A06 to Kyoto University). We are grateful to Eliza- beth Nakajima for her reading an earlier version of this manuscript and correcting its English.

\section{References}

Baddeley, A. D. (2000). The episodic buffer: A new component of working memory. Trends in Cognitive Sciences, 4, 417-423. doi:10.1016/S1364-6613(00)01538-2

Bull, H., Marshark, M., \& Baltto-Vallee, F. (2005) SNARC hunting: Examining number representation in deaf students. Learning and Individual Differences, 15, 223-236. doi:10.1016/j.lindif.2005.01.004

Dehaene, S. (1997). Number sense. Oxford: Oxford University Press.

Deahaene, S., Dupoux, E. \& Mehler, J. (1990). Is numerical comparison digital? Analogical and symbolic effects in two-digit number comparison. Journal of Experimental Psychology, Human Perception and Performance, 16, 626-641. doi:10.1037/0096-1523.16.3.626

Dehaene, S., Piazza, M., Pinel, P. \& Cohen, L. (2003). Three parietal circuits for number processing. Cognitive Neuroscience, 20, 487-506.

Frosted, P. (1996). Mathematical achievement of hearing-impaired students in Norway. European Journal of Special Needs of Education, 11, 67-81.

Gallistel, C. R., \& Gelman, R. (1992). Preverbal and verbal counting and computation. Cognition, 44, 43-74. doi:10.1016/0010-0277(92)90050-R

Heiling, K. (1995). The development of deaf school children. Hamburg: Signum.

Lancy D. F (1983). Cross-cultural studies in cognition and arithmetic. New York, NY: Academic Press.

Masataka, N., Ohnishi, T., Imabayashi, E., HIrakara, M., \& Matsuda, H. (2007). Neural correlates for learning to read Roman numerals. Brain and Language, 100, 276-282. doi:10.1016/j.bandl.2006.11.011

McComb, K., Packer, C., \& Pusey, A. (1994). Roaring and numerical assessment in contests between groups of female lions, Panthara leo. Animal Behaviour, 47, 379-387. doi:10.1006/anbe.1994.1052

Nunes, T. \& Moreno, C. (2002). An intervention program to promote deaf pupils' achievement in mathematics. Journal of Deaf Studies and Deaf Education, 7, 120-133. doi:10.1093/deafed/7.2.120

Paas, F., Renkl, A. \& Sweller, J. (2003). Cognitive load theory and instructional design: Recent developments. Educational Psychologist, 38, 1-4. doi:10.1207/S15326985EP3801 1

Piazza, M., \& Dehaene, S. (2005) From number neurons to mental arithmetic: The cognitive neuroscience of number sense. In M. S. Gazzaniga (Ed.), The Cognitive neurosciences III (pp. 865-875) Cambridge, MA: MIT press.

Sousa, D. A. (2008) How the brain learns mathematics. Thousand Oaks, CA: Corwin.

Sweller, J. (1988). Cognitive load during problem solving: Effects on learning. Cognitive Science, 12, 257-285. doi:10.1207/s15516709cog1202_4 